\title{
Bayesian game model to analyze electric vehicles and smart grid interaction
}

\author{
Saeed Nejadfard Jahromi ${ }^{\text {a }}$, Alireza Hamedi ${ }^{a}$, Mohammad Bagher Ahmadi ${ }^{\text {b }}$ \\ Mohammad Mohammadi ${ }^{\mathrm{a}}$ \\ a Department of Power and Control Engineering, Shiraz University, Shiraz 7196484334, Iran \\ ${ }^{\mathrm{b}}$ Department of Mathematics, Shiraz University, Shiraz 7196484334, Iran
}

\begin{abstract}
In this paper, a novel Bayesian game model is developed to estimate the behavior of the Electric Vehicles (EVs) and smart grid. The proposed model focus on EVs as consumers and how to combine them efficiently into the smart grid. We first briefly introduce the smart grid and its structure. Then, the proposed model is presented and the effect of different variables such as load demand, energy price, and battery charge are investigated. Finally, the simulation is carried out on a test case and the obtained results demonstrate that our models help to efficiently combine the EVs into the smart grid.
\end{abstract}

Keywords: smart grid, Bayesian game, electric vehicles.

\section{Introduction}

A smart grid is defined as an intelligent power network that combines various technologies in power and communication to monitor and optimize the operations of all functional units from electricity generation to end-customers[1]. The large penetration of EVs, if properly managed, can support the electricity smart distribution network. EVs batteries' can be utilized as the storage devices to mitigate the fluctuations in power demand and providing frequency control [2]. Smart grids should have a good energy structure allowing it to deliver electricity efficiently with minimal losses. Moreover, the need of a distributed communication network seems to be fundamental to guarantee a bidirectional flow of information between all the smart grid actors. The emergence of EVs in the power grid has created a fear of falling into the electricity frequency due to the huge number of vehicles, which can be deployed in the grid [3]. These latter are considered to be one of the major consumers of electricity in smart grid [4]. As a consequence, analysing the effect of EVs charging on smart grid and designing an optimal charging strategy for EVs are important. In [3], the authors proposed a non-cooperative Stackelberg game model to optimize the combination of EVs into the smart grids. In [5], the authors developed a mean field model to show the behaviour of EVs and plug hybrid electric vehicle (PHEV) owners aiming selfishly to maximize their satisfaction under electricity pricing policy constraints. A novel algorithm based on cooperative game theory was developed for the integrated wind turbines power system to optimize the wind turbines and power system profits [6]. A non-cooperative game model is presented in [7] to survey energy trading in the smart grid. In this paper, we first define a smart grid architecture and the combination of EVs into the smart grids briefly. Then, we develop a game model that aims to optimize the charging of EVs. This model is based on Bayesian Nash Equilibrium (BNE), which aims to make a balance between the electricity offer and demand. The novelty introduced in this paper compared with the other works is a

\footnotetext{
* Manuscript received January 10, 2018; revised April 12, 2018.

Corresponding author.E-mail address: a.hamedi@shirazu.ac.ir.

doi: $10.12720 /$ sgce.7.2.125-129
} 
theoretic game approach considering decisive parameters which proposed to combine optimally EVs in the smart grid.

\section{Smart Grid Architecture}

In this section, we introduce the architecture of the smart grid that takes into account the existing equipment and applications to ensure the proper functioning of this network. Moreover, with a good vision of this architecture, power system stockholders can predict the future requirements. The most challenging issue on the smart grid is to optimize the management of its resources and equipment in order to cover the average demand. The power network has as goal to distribute electricity to all users. Due to the excessive increasing of the electricity demand caused by diverse factors such as the birth of new and diverse consumers like the EVs, the smart grid has to promote its capacity and increase its production by deploying decentralized and fluctuant energy sources such as wind and solar power [8-10]. All this requires a new and complete vision of the electricity network to quickly overcome peak demand of electricity and intermittency of energy sources. The current vision of an effective smart grid offers a great opportunity to the modernization of public services through a vast increase in number and types of connected devices. This migration to a smart network using connected devices is driving the need for an increased bandwidth network with a high safety and reliability. So, there is a need for a real study to establish the necessary infrastructure to ensure a good two-way communication between the different stakeholders and to exchange useful information such as price, availability, and amount of required electricity. The proposed smart grid architecture to analyze the interaction between smart grid and EVs is depicted in Figure. 1.

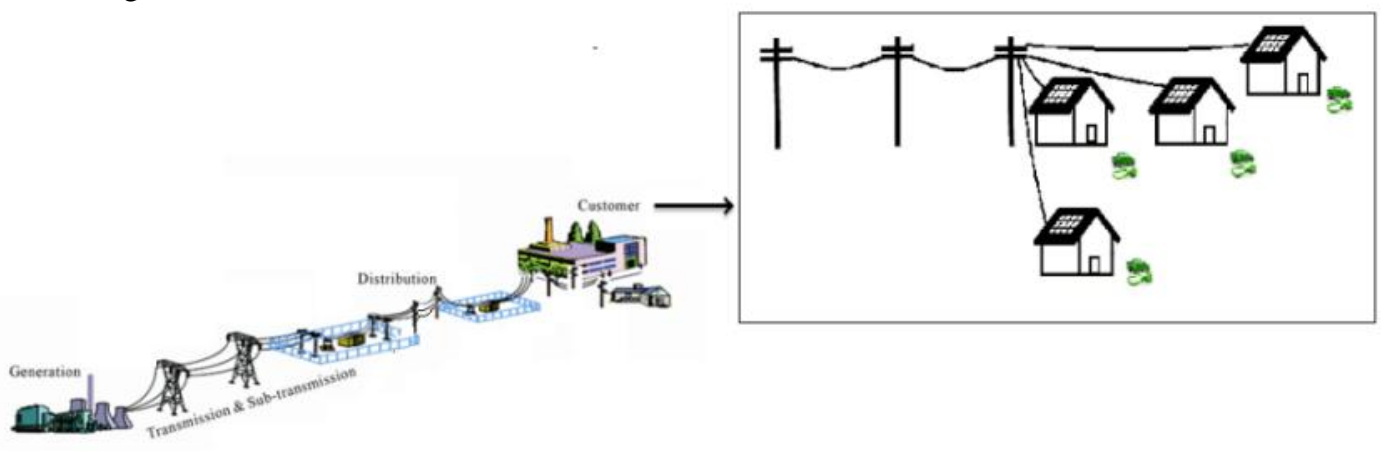

Fig. 1. EVs and smart grid interaction

As can be seen, EVs can be charged either from the smart grid or from other alternative sources such as rooftop photovoltaic panels.

\section{Game Formulation}

In this section, we define the Bayesian game model to combine the EVs into the smart grid. We first provide the payoff matrix of the game related to our two players, which are: the SG as an electricity supplier and the EV as a consumer. Afterward, we define a set of strategies that could occur between these players. Finally, based on these strategies and with the help of BNE concept, we predict the action of each player.

A Bayesian game can be described by $\left\langle N,\left\{A_{i}\right\}_{i=1}^{n},\left\{\Theta_{i}^{n}\right\}_{i=1}^{n},\left\{v_{i}\left(. ; \theta_{i}\right), \theta_{i} \in \Theta_{i}\right\}_{i=1}^{n},\left\{\phi_{i}\right\}_{i=1}^{n}\right\rangle$ where N is the set of players, $A_{i}$ indicates the action set of player $i, \Theta_{i}$ describes the type space player $i, v_{i}$ indicates the payoff of the $i$ th player and $\phi_{i}$ is the belief of player $i$ with respect to the uncertainty over the other players' types. In our work, we consider two players: the SG and the EV; $N=\{S G, E V\}$. Each player chooses to perform a specific action in order to maximize its payoff. We note that, in game theory concept, players are rational and want to maximize their own payoff. The SG can perform one of these two pure 
strategies: deliver or do not deliver; $\theta_{S G}=\{d, d d\}$. The EVs also have two type: charging or do not charging; $\theta_{E V}=\{c, d c\}$.

The strategies can be described mathematically as follows: $S_{1}\left(\theta_{1}=d\right)=D, S_{1}\left(\theta_{1}=d d\right)=D D$ and $S_{2}\left(\theta_{2}=c\right)=C, S_{2}\left(\theta_{2}=d c\right)=D C$. The "Nature" randomly chooses a type for each player based on the probability distribution across the each player's type space. Let $\mathrm{q}_{1}$ be the prior probability of player SG being deliver type $\theta_{S G}=d$ and therefore $\theta_{S G}=d d$ is equal to $1-\mathrm{q}_{1}$. Similarly, Let $\mathrm{p}_{1}$ be the prior probability of player EV being charge type $\theta_{E V}=c$ and therefore $\theta_{E V}=d c$ is equal to $1-\mathrm{p}_{1}$. It should be noted that $\mathrm{p}_{1}, 1-\mathrm{p}_{1}, \mathrm{q}_{1}$ and $1-\mathrm{q}_{1}$ are common knowledge. In this game, we divide time into regular intervals called time-slots; each one of them represents a stage. However, at each stage there is an interaction between the SG and an EV. Therefore, with the help of Bayesian game concept, we model the different strategies that could occur between an EV and the SG. The future state prediction for the behavior of each one is determined by the BNE. The extensive form of the game is depicted in the Figure. 2.

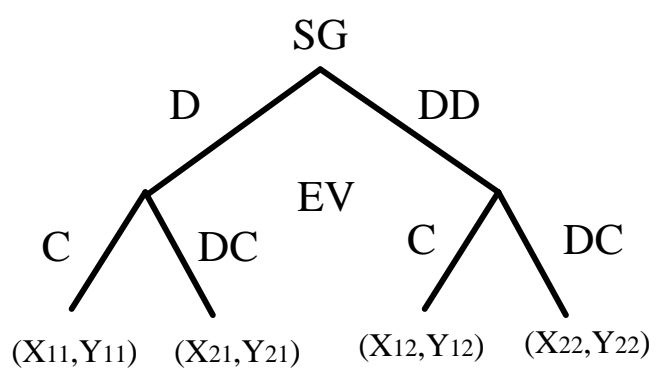

Fig. 2. Extensive form of the game

Table 1. Presents the prior belief. The payoff equation can be described as follows:

$$
\begin{array}{ll}
X_{11}=P(t) \cdot\left(C_{\text {rev }}(t)-C_{\text {pur }}(t)\right) ; & Y_{11}=\operatorname{soc}(t)-C_{\text {rev }}(t) \cdot P(t)+P_{\text {driv }}(t) ; \\
X_{12}=\operatorname{soC}(t) \cdot C_{\text {pur }}(t) ; & Y_{12}=\operatorname{soc}(t) ; \\
X_{21}=0 ; & Y_{21}=\operatorname{soc}(t)+\left(P_{\text {driv }}(t)-1\right) \\
X_{22}=0 ; & Y_{22}=\operatorname{soc}(t)
\end{array}
$$

Where $P(t)$ is the amount of requested electricity at the time $t, C_{\text {rev }}(t)$ and $C_{\text {pur }}(t)$ are the revenue obtained from the selling energy in time $t$ and the cost of purchased energy at time $t$ simultaneously. $\operatorname{SOC}(t)$ is also the state of charge of the battery of EV and $P_{\text {driv }}(t)$ is the requiring power for driving purpose. It should be noted that all payoffs are normalized in order to create dimensionless variables. Therefore, each payoff equals to a score without unit. For example, the amount of requested electricity $\mathrm{P}(\mathrm{t})$ is normalized as follows: $\left(\mathrm{P}(\mathrm{t})=\mathrm{P}_{\text {real }} / \mathrm{P}_{\max }\right)$.

Table 1. Prior belief

\begin{tabular}{|c|c|c|}
\hline SG & $C$ & $D C$ \\
\hline$D$ & $p_{1} q_{1}$ & $q_{1}\left(1-p_{1}\right)$ \\
\hline$D D$ & $p_{1}\left(1-q_{1}\right)$ & $\left(1-p_{1}\right)\left(1-q_{1}\right)$ \\
\hline
\end{tabular}




\subsection{Equilibrium determination}

According to Nash, there is a mixed strategy $B N E\left\{S_{S G}\left(A_{S G}, q^{*}\right), S_{E V}\left(A_{E V}, p^{*}\right)\right\}$ in which both players do not change their actions. Therefore, the BNE concept is utilized to predict the stage that the SG and the EV do not change their strategies, which are delivered and charge, respectively. Consequently, there is a mixed strategy $\mathrm{BNE}\left\{\mathrm{S}_{\mathrm{SG}}\left(\right.\right.$ deliver, $\left.\mathrm{q}_{1} *\right), \mathrm{S}_{\mathrm{EV}}\left(\right.$ charge, $\left.\left.\mathrm{p}_{1} *\right)\right\}$ so that the $\mathrm{SG}$ chooses to deliver action when the probability $\mathrm{q}_{1}>\mathrm{q}_{1} *$ and the EV chooses charge action when $\mathrm{p}_{1}>\mathrm{p}_{1} *$. The proof of this theorem is presented as follows:

- $U_{E V}($ charge $)=p_{1} \cdot Y_{11}+\left(1-p_{1}\right) \cdot Y_{21}=\operatorname{SOC}(t)+P_{\text {driv }}(t)-1+p_{1}-p_{1} C_{\text {rev }}(t) \cdot P(t)$

- $U_{E V}\left(\right.$ do not charge $=p_{1} \cdot Y_{12}+\left(1-p_{1}\right) \cdot Y_{22}=\operatorname{soC}(t)$

It is clear that EV select charge action when $\mathrm{U}_{\mathrm{EV}}$ (Charge) $>\mathrm{U}_{\mathrm{EV}}$ (Do not charge). This situation can describe as follows:

- $p_{1}>p_{1}^{*} \quad$ where $p_{1}^{*}=\frac{1-P_{\text {driv }}(t)}{1-C_{r e v}(t) \cdot P(t)}$ with $0<p_{1}^{*}<1$

Similar to EVs, the mixed strategy of the SG can be defined as follows:

- $U_{S G}($ deliver $)=q_{1} \cdot X_{11}+\left(1-q_{1}\right) \cdot X_{12}=q_{1} \cdot P(t) \cdot\left(C_{\text {rev }}(t)-C_{\text {pur }}\right)+\left(1-q_{1}\right) \cdot \operatorname{SOC}(t) \cdot C_{\text {pur }}(t)$

- $U_{S G}$ (do not deliver $=q_{1} \cdot X_{21}+\left(1-q_{1}\right) \cdot X_{22}=0$

The $\mathrm{SG}$ will choose to deliver action when $\mathrm{U}_{\mathrm{SG}}$ (Deliver) $>\mathrm{U}_{\mathrm{SG}}$ (Do not deliver). This situation can describe as follows:

- $q_{1}<q_{1}^{*} \quad$ where $q_{1}^{*}=\frac{\operatorname{soc}(t) \cdot C_{p u r}(t)}{\operatorname{soC}(t) \cdot C_{p u r}(t)+P(t)\left(C_{p u r}(t)-C_{r e v}(t)\right)}$ with $0<q_{1}^{*}<1$

Thus, we can conclude that the Nash equilibrium corresponds to $\left(\mathrm{q}_{1}{ }^{*}, \mathrm{p}_{1}{ }^{*}\right)$ and in this situation, no one wants to change related actions.

\section{Simulation Results}

The effect of load demand and selling energy price are tested in this paper. The load demand, energy price, and SOC data are gathered from [11, 12]. The simulation is carried out for 24 hours and the load is categorized to three periods, peak time, mid-time and off-peak time. The load demand and energy price are presented in Figure.3 (a) and Figure.3 (b) respectively.
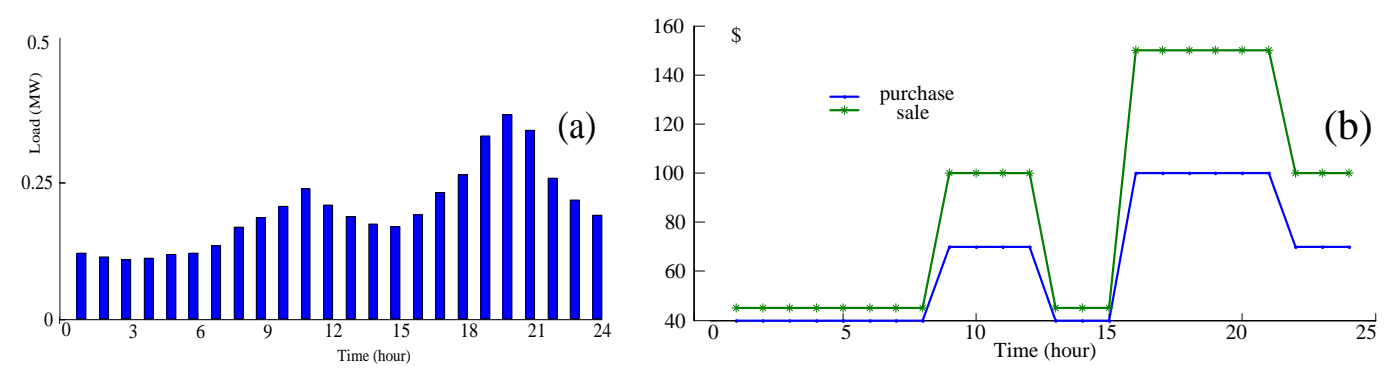

Fig. 3. The load demand and energy price

It should be noted that energy price is related to load demand such that as the load demand increases the energy price is also increases. Several states are considered and different $\mathrm{SOC}, P_{\text {driv }}$ and $P(t)$ are considered and the result are depicted in Figure. 4.

As can be seen in the figure. $4, p_{1}^{*}$ is increasing as the load increases. This is due to the fact that when the load increases the cost of energy is also increasing. Therefor the EVs are willing to purchase energy in 
off-peak or mid-peak. On the other hand $q_{1}^{*}$ is decreasing from off-peak time to peak time. Thus, SG is willing to sale its energy in off-peak time.

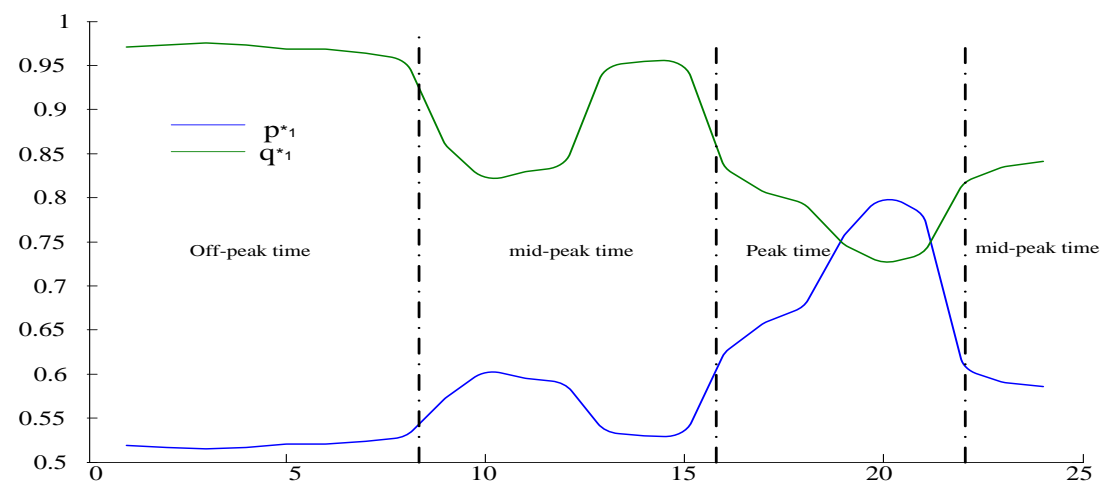

Fig. 4. simulation results

\section{Conclusion}

During the past years, the smart grids confront many changes, in addition, fundamental changes. But they still need more improvement because of the nonstop birth of new requirements and challenges. In this paper, we proposed Bayesian game models to combine EVs into the SG. Simulation results show the efficiency and good performance of the proposed model. To achieve more realistic simulation results, we considered three different time periods: off-peak, mid-peak and on-peak times to ensure more accurately the balance between the electricity demand and supply. Therefore, the proposed model offer a good management of electricity flow through a good operation of resources. The obtained simulation results demonstrate that our models help to efficiently combine the electric vehicles into the smart grid.

\section{References}

[1] W. Meng, R. Ma, and H.-H. Chen, "Smart grid neighborhood area networks: a survey," IEEE Network, vol. 28, no. 1, pp. 2432, 2014.

[2] M. Singh, P. Kumar, and I. Kar, "A multi charging station for electric vehicles and its utilization for load management and the grid support," IEEE Transactions on Smart Grid, vol. 4, no. 2, pp. 1026-1037, 2013.

[3] W. Tushar, W. Saad, H. V. Poor, and D. B. Smith, "Economics of electric vehicle charging: A game theoretic approach," IEEE Transactions on Smart Grid, vol. 3, no. 4, pp. 1767-1778, 2012.

[4] W. Lee, L. Xiang, R. Schober, and V. W. Wong, "Electric vehicle charging stations with renewable power generators: A game theoretical analysis," IEEE Transactions on Smart Grid, vol. 6, no. 2, pp. 608-617, 2015.

[5] R. Couillet, S. M. Perlaza, H. Tembine, and M. Debbah, "A mean field game analysis of electric vehicles in the smart grid," in Computer Communications Workshops (INFOCOM WKSHPS), 2012 IEEE Conference on, 2012, pp. 79-84: IEEE.

[6] E. Baeyens, E. Bitar, P. P. Khargonekar, and K. Poolla, "Wind energy aggregation: A coalitional game approach," in Decision and Control and European Control Conference (CDC-ECC), 2011 50th IEEE Conference on, 2011, pp. 3000-3007: IEEE.

[7] Y. Wang, W. Saad, Z. Han, H. V. Poor, and T. Başar, "A game-theoretic approach to energy trading in the smart grid," IEEE Transactions on Smart Grid, vol. 5, no. 3, pp. 1439-1450, 2014.

[8] R. C. Green, L. Wang, and M. Alam, "The impact of plug-in hybrid electric vehicles on distribution networks: A review and outlook," Renewable and sustainable energy reviews, vol. 15, no. 1, pp. 544-553, 2011.

[9] W. Su, H. Eichi, W. Zeng, and M.-Y. Chow, "A survey on the electrification of transportation in a smart grid environment," IEEE Transactions on Industrial Informatics, vol. 8, no. 1, pp. 1-10, 2012.

[10] C. Vineetha and C. Babu, "Smart grid challenges, issues and solutions," in Intelligent Green Building and Smart Grid (IGBSG), 2014 International Conference on, 2014, pp. 1-4: IEEE.

[11] F. Rassaei, W.-S. Soh, and K.-C. Chua, "Demand response for residential electric vehicles with random usage patterns in smart grids," IEEE Transactions on Sustainable Energy, vol. 6, no. 4, pp. 1367-1376, 2015.

[12] M. E. Hajiabadi and H. R. Mashhadi, "Analysis of the Probability Distribution of LMP by Central Limit Theorem," IEEE Transactions on Power Systems, vol. 28, no. 3, pp. 2862-2871, 2013. 